\title{
Epidemiological Survey and Phylogenetic Characterization of Cysticercus tenuicollis Isolated from Tibetan Pigs in Tibet, China
}

\author{
Houqiang Luo, ${ }^{1,2}$ Hui Zhang, ${ }^{1}$ Kun Li, ${ }^{1}$ Mujeeb Ur Rehman, ${ }^{1}$ Khalid Mehmood, ${ }^{1,3}$ \\ Yanfang Lan, ${ }^{1}$ Shucheng Huang, ${ }^{1}$ and Jiakui $\mathrm{Li}^{1,4}$ \\ ${ }^{1}$ College of Veterinary Medicine, Huazhong Agricultural University, Wuhan, China \\ ${ }^{2}$ College of Animal Science, Wenzhou Vocational College of Science \& Technology, Wenzhou, China \\ ${ }^{3}$ University College of Veterinary \& Animal Sciences, The Islamia University of Bahawalpur, Bahawalpur, Pakistan \\ ${ }^{4}$ Tibet Agriculture and Animal Husbandry College, Linzhi, Tibet, China \\ Correspondence should be addressed to Jiakui Li; lijk210@sina.com
}

Received 2 January 2017; Revised 29 March 2017; Accepted 26 April 2017; Published 18 May 2017

Academic Editor: Jacques Cabaret

Copyright (c) 2017 Houqiang Luo et al. This is an open access article distributed under the Creative Commons Attribution License, which permits unrestricted use, distribution, and reproduction in any medium, provided the original work is properly cited.

\begin{abstract}
Cysticercus tenuicollis, commonly known as "water bell," is a larva of Taenia hydatigena, which is the most significant parasite of pigs. However, until now very few information is available regarding the prevalence and genetic characterization of the Cysticercus tenuicollis in Tibetan pigs. Therefore, the aim of this study was to investigate the prevalence and phylogenetic analysis of Cysticercus tenuicollis in Tibetan pigs. For this purpose, the COX2 gene of Cysticercus tenuicollis was amplified and sequenced for the first time in Tibetan pigs. The overall prevalence of Cysticercus tenuicollis was $43.93 \%$ in Tibetan pigs, with further distribution of $42.86 \%$ in 2014 and $45.35 \%$ in 2015. In Tibetan male and female pigs, the prevalence of Cysticercus tenuicollis was $43.39 \%$ and $44.56 \%$, respectively. The prevalence of Cysticercus tenuicollis in different growing stages (juveniles, subadults, and adults) varied from 30.20\% to $63.79 \%$. The phylogenetic analysis of the Cysticercus tenuicollis isolates showed very close resemblance to 16 reference strains, isolates from Gansu, Hunan, and Sichuan provinces of China. To the best of our knowledge, this is the first report on the prevalence and genetic characterization of Cysticercus tenuicollis derived from Tibetan pigs. The data of present study provides baseline information for controlling cysticerci infections in pigs in Tibetan Plateau, China.
\end{abstract}

\section{Introduction}

Cysticercus tenuicollis is a bubble-like metacestode of Taenia hydatigena that invades the abdominal cavity and liver of pigs, dogs, and other carnivores [1-3]. This parasite is generally associated with the significant tissue damage in liver, lungs, and other organs of pigs, cattle, sheep, and other types of livestock [4-7]. The parasite can infect the pigs of any age and can not only shorten the feed intake but also hinder the growth and development of animals. Therefore, considerable tissue damage of liver or other organs by this parasite may perhaps lead to the death or serious economic losses to the pig industry [8].

The prevalence of Cysticercus tenuicollis in domestic pigs has been reported from certain parts of China. Previously, a low prevalence of Cysticercus tenuicollis (1.08\%) in pigs was reported from Hunan province of China, whereas the high prevalence rates (42.43\% to 35.98\%) were observed in Fujian and Yunnan [9-11]; additionally, regional distribution of Cysticercus tenuicollis infection in pigs has been also reported from Sichuan and Yunnan [12]. However, no report has been available about the prevalence of Cysticercus tenuicollis infection in Tibetan pigs.

Tibetan pigs are relatively ancient original indigenous breed, a rare plateau type of pigs in the world, and are the only high altitude pasture pigs in China. Tibetan pigs are mainly distributed in the Qinghai-Tibetan Plateau in China (Qinghai, Sichuan, Yunnan, and eastern Tibet region) [13]. With high proteins and rich amino acids, the Tibetan pig meat is an important source of income for Tibetans nomads [13]. Due to outdoor grazing of Tibetan pigs round the year with the main intermediate hosts (domestic dogs and pi-dog) of the 


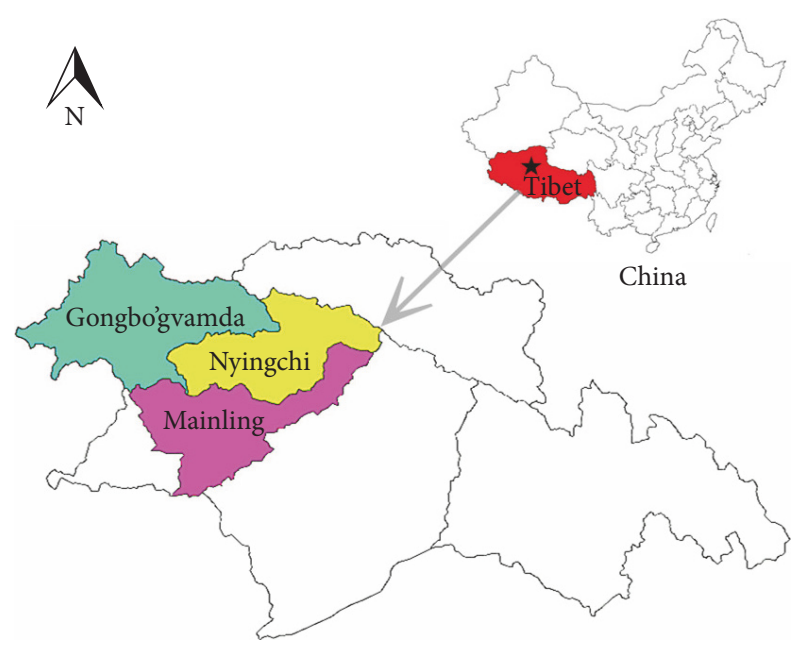

FIGURE 1: The map of geographical distribution of Cysticercus tenuicollis serological investigation in Tibet.

Cysticercus tenuicollis, pigs can be exposed to this infection. Furthermore, due to the lack of knowledge at herdsmen level and no special protection, awareness or insect repellent program of the parasitic infection in study area enhances the risk of this infection in ancient original indigenous breed and can lead to significant economic losses.

To date, no report is available about the prevalence and genetic characterization of Cysticercus tenuicollis in Tibetan pigs, China. The current study was aimed at investigating the prevalence and genetic characterization of cytochrome oxidase subunit 2 (COX2) derived from Cysticercus tenuicollis in Tibetan pigs.

\section{Materials and Methods}

2.1. Study Site. This study was conducted in three counties (Nyingchi, Mainling, and Gongbo'gvamda) of Tibet, China, and samples were collected from pigs at different slaughterhouses in these three counties that have an average elevation of 3100 meters with the largest continuous high elevation ecosystem (Figure 1).

2.2. Samples Collection and Processing. In present study, a total of 112 samples were collected from Tibetan pigs (independent of age, sex, and breed by using simple random technique for sample collection) at different slaughterhouses of Nyingchi, Mainling, and Gongbo'gvamda of Tibet, in 2014. The information regarding each sample was captured on a prescribed performa. Similarly, a total of 86 samples were also collected from these counties in 2015. After autopsy, each positive sample was recorded and collected (Figure 2). The larval stages were washed extensively in $0.9 \%$ sodium chloride solution and identified through morphological examinations. Subsequently, they were fixed in $75 \%$ alcohol $(\mathrm{V} / \mathrm{V})$ and stored at $-20^{\circ} \mathrm{C}$, until subsequent use and further analysis. Total DNA of the larvae of Cysticercus tenuicollis was extracted using the commercial kit (TIANamp Genomic

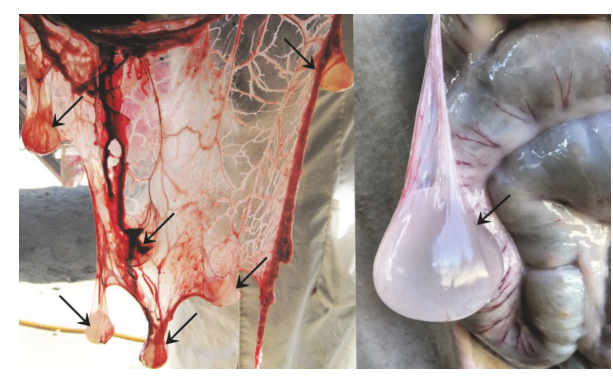

FIgURE 2: Cysticercus tenuicollis infection in clinical autopsy in Tibetan pigs, China.

DNA Kit, TianGen, China), according to manufacturer's instructions. The eluted DNA was stored at $-20^{\circ} \mathrm{C}$ until further use.

A PCR amplification approach was used to amplify a fragment $(\sim 560 \mathrm{bp})$ of the COX2 gene. For this purpose polymerase chain reaction primer forward GAAGTTGGTTACTGAAAAG and reverse ATTCCATGATTAACACCA$\mathrm{C}$ were used with the following cycling parameters: 35 cycles at $95^{\circ} \mathrm{C}$ for 30 seconds and $72^{\circ} \mathrm{C}$ for 1 minutes, annealing at $55^{\circ} \mathrm{C}$ for 30 seconds, and final extension at $72^{\circ} \mathrm{C}$ for 5 minutes. $\mathrm{ddH}_{2} \mathrm{O}$ was used as blank control group. PCR products were separated on agarose gel (1.0\%) along with ethidium bromide (at the rate of $0.5 \mu \mathrm{g} / \mathrm{ml}$ ) and electrophoresis was performed on $0.5 \mathrm{x}$ TBE buffer at $5 \mathrm{~V} / \mathrm{cm}$ for 60 minutes. The products were purified using a TaKaRaMiniBEST Agarose Gel DNA Extraction Kit Ver.4.0 (Takara Biotechnology Co., Ltd., Dalian, China), according to manufacturer's instructions. The obtained positive products were sequenced by a commercial company (Quintara Biosciences, Wuhan, China).

2.3. Phylogenetic and Sequence Analysis. The nucleotide sequences of the COX2 gene of Cysticercus tenuicollis were compared with previously reported Cysticercus tenuicollis sequences available at NCBI database (appears in Table 2). Multiple alignments and phylogenetic analysis were conducted using Molecular Evolutionary Genetic Analysis (MEGA 6.0) software. Phylogenetic tree was constructed using the neighbor-joining method. The evolutionary distances were estimated using the Kimura two-parameter method. A bootstrapping test was performed with 1000 duplicates and the transversion/transition rate was set at 2.0.

2.4. Statistical Analysis. The prevalence of Cysticercus tenuicollis in Tibetan pigs relating to different genders and growing stages was analyzed statistically by using Chi-square test, while 95\% CI and odd ratio were also determined. The value of $P<0.05$ was considered as statistically significant.

\section{Results}

3.1. Prevalence of Cysticercus tenuicollis. The results showed the highest infection rate of Cysticercus tenuicollis in Tibetan pigs was $43.93 \%$ in all three counties. The year wise prevalence of Cysticercus tenuicollis was $42.86 \%$ and $45.35 \%$ in 2014 


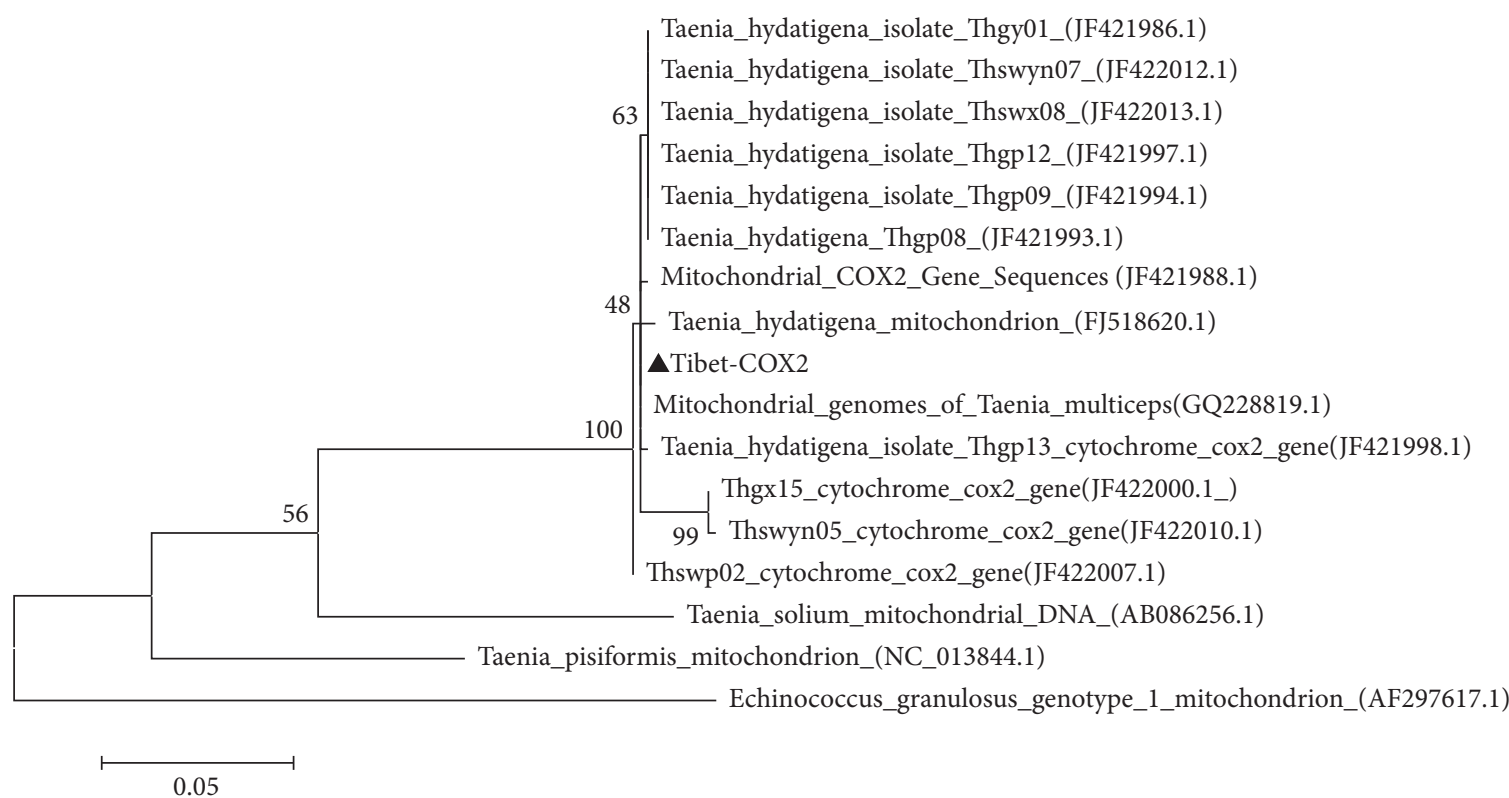

FIgure 3: Phylogenetic tree constructed by the neighbor-joining method in MEGA 6.0, using nucleotide sequences of the COX2 gene (Outgroup AF297617.1).

and 2015, respectively. The sex wise prevalence of Cysticercus tenuicollis was $42.37 \%$ and $44.68 \%$ in male and $46.15 \%$ and $43.40 \%$ in female pigs in 2014 and 2015, respectively, while during different growing stages of Tibetan pigs, the prevalence was $29.51 \%, 60.00 \%$, and $56.25 \%$ in 2014 and $31.43 \%$, $69.57 \%$, and $42.86 \%$ in 2015 in juveniles, subadults, and adults, respectively. The rate of infection among males and females was not found statistically significantly $(P>0.05)$; however, a significant difference $(P<0.05)$ was observed among different growing stages of Tibetan pigs as shown in Table 1.

3.2. Phylogenetic Analysis. To determine the evolutionary relationships between the larvae stage of Cysticercus tenuicollis (named: Tibet-COX2) and previously reported 16 reference strains, the result revealed that the nucleotide sequence of the Tibet-COX2 strain was $97.7 \%-100 \%$ identical to that of the previously reported strains (accession numbers GQ228819.1, JF421988.1, JF421998.1, JF422007.1, FJ518620.1, JF422000.1, JF421986.1, JF422012.1, JF421997, JF422013.1, JF421994.1, JF421993.1, JF422010.1, and AB086256.1, NC013844.1, and AF297617.1) (Table 2). The phylogenetic analysis was performed based on the COX2 gene (Figure 3 ). As determined by the phylogenetic tree, the homology of the nucleotide sequence between the Tibet-COX2 strain and some reference strains (GQ228819.1, JF421988.1, JF421998.1, JF422007.1, FJ518620.1, JF422000.1, JF421986.1, JF422012.1, JF421997, JF422013.1, JF421994.1, JF421993.1, and JF422010.1) illustrated a close homology. The result showed that the TibetCOX2 strain was very closely related to reference strains isolated from Gansu, Hunan, and Sichuan provinces, which are very close regions to Tibet (Figure 3 ). In addition, the nucleotide sequences of isolated strains shared $97.7 \%-100 \%$ sequence identify with the reference strains (Figure 4).

\section{Discussion}

Cysticercus tenuicollis is the larvae stage of Taenia hydatigena, which is a common parasite of pigs, cattle, buffalo, yak, sheep, goat, camel, horse, and human [6]. Many countries in the world have reported Cysticercus tenuicollis since its first report $[4,6,7]$. This disease remains a severe problem in pigs especially under the age of one year, where there are no effective measures that result in very high mortality. China is the largest pigs producing country, while parasitosis has become a major threat to pigs industry in the country, which causes serious illness [13]. As the only plateau and alpine pasture of pig breeds in China, Cysticercus tenuicollis has not yet been reported in Tibetan pigs. The recent research showed that a high infection rate of Cysticercus tenuicollis was found in Tibetan pigs, which is higher than that reported in pigs from Dali $(6.06 \%)$ and Pingle $(1.11 \%)$ [13, 14]. The prevalence difference of Cysticercus tenuicollis in our study and the previous reports may be due to the differences in freerange feeding pattern, ecological environment, and existence of the wild dogs, as well as the knowledge level of the breeder [15]. During the survey, we found that the local people were not aware of using antiparasitic drugs, secondly improperly or frequently using the antimicrobial agents. So, it may be the possible reason for the high prevalence rate of Cysticercus tenuicollis. Tibetan pigs are mainly fed by the extensive breeding environment combined with the dry lot husbandry [16]. Under the special breeding system, this animal get more chance to expose to the external environment; among them, subadults and adults have the longest time of outdoor activities which increases a higher infection risk of infecting the Cysticercus tenuicollis, compared with the juveniles Tibetan pigs [6]. 


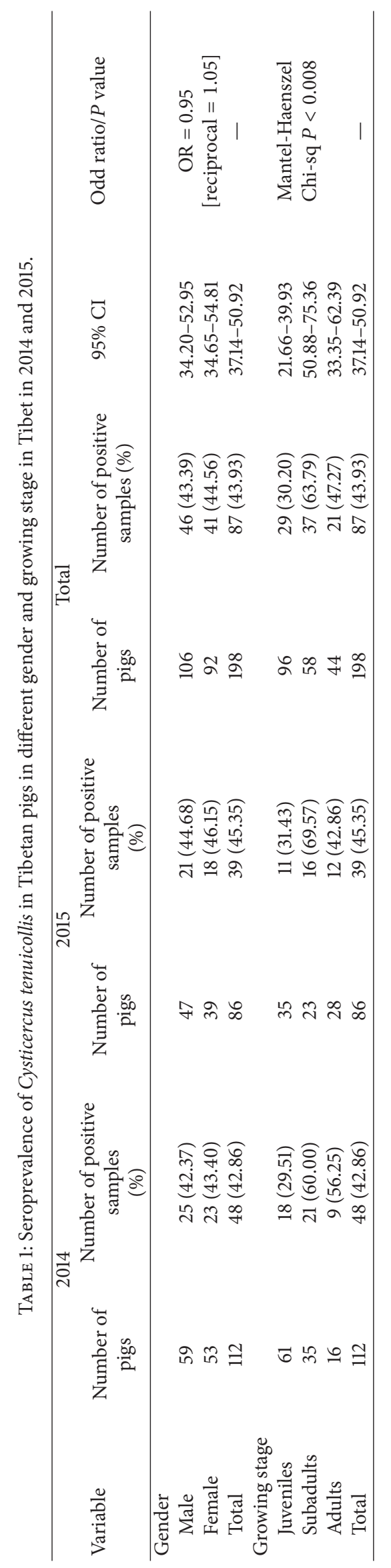


TABLE 2: The data obtained from NCBI for analysis in this study.

\begin{tabular}{|c|c|c|c|c|}
\hline Accession & Regions & Year & Species & Strain \\
\hline GQ228819.1 & Gansu & 2010 & Sheep & $\mathrm{IU}$ \\
\hline JF421988.1 & Sichuan & 2012 & Goat & Thgj03 \\
\hline JF421998.1 & Sichuan & 2012 & Goat & Thgp13 \\
\hline JF422007.1 & Sichuan & 2012 & Pig & Thswp02 \\
\hline FJ518620.1 & Hunan & 2011 & Human & IU \\
\hline JF422000.1 & Sichuan & 2012 & Goat & Thgx15 \\
\hline JF422010.1 & Sichuan & 2012 & Pig & Thswyn05 \\
\hline JF421993.1 & Sichuan & 2012 & Goat & Thgp08 \\
\hline JF421994.1 & Sichuan & 2012 & Goat & Thgp09 \\
\hline JF421997.1 & Sichuan & 2012 & Goat & Thgp12 \\
\hline JF422013.1 & Sichuan & 2012 & Pig & Thswx08 \\
\hline JF421986.1 & Sichuan & 2012 & Goat & Thgy01 \\
\hline JF422012.1 & Sichuan & 2012 & Pig & Thswyn07 \\
\hline
\end{tabular}

\begin{tabular}{|c|c|c|c|c|c|c|c|c|c|c|c|c|c|c|c|c|c|}
\hline & \multicolumn{17}{|c|}{ Percent identity } \\
\hline & & 1 & 2 & 3 & 4 & 5 & 6 & 7 & 8 & 9 & 10 & 11 & 12 & 13 & 14 & & \\
\hline & 1 & & 99.6 & 100.0 & 99.8 & 99.8 & 99.8 & 98.3 & 98.1 & 99.8 & 99.8 & 99.8 & 99.8 & 99.8 & 99.8 & 1 & Tibet-COX2 \\
\hline & 2 & 0.4 & & $99.6=$ & 99.4 & 99.4 & 99.4 & 97.9 & 97.7 & 99.4 & 99.4 & 99.4 & 99.4 & 99.4 & 99.4 & 2 & FJ518620.1 \\
\hline & 3 & 0.0 & 0.4 & & 99.8 & 99.8 & 99.8 & 98.3 & 98.1 & 99.8 & 99.8 & 99.8 & 99.8 & 99.8 & 99.8 & 3 & GQ228819.1 \\
\hline & 4 & 0.2 & 0.6 & 0.2 & & 99.6 & 99.6 & 98.1 & 97.9 & 99.6 & 99.6 & 99.6 & 99.6 & 99.6 & 99.6 & 4 & JF421988.1 \\
\hline & 5 & 0.2 & 0.6 & 0.2 & 0.4 & & 99.6 & 98.1 & 97.9 & 99.6 & 99.6 & 99.6 & 99.6 & 99.6 & 99.6 & 5 & JF421998.1 \\
\hline & 6 & 0.2 & 0.6 & 0.2 & 0.4 & 0.4 & & 98.1 & 97.9 & 99.6 & 99.6 & 99.6 & 99.6 & 99.6 & 99.6 & 6 & JF422007.1 \\
\hline & 7 & 1.7 & 2.1 & 1.7 & 1.9 & 1.9 & 1.9 & & 99.8 & 98.1 & 98.1 & 98.1 & 98.1 & 98.1 & 98.1 & 7 & JF422000.1 \\
\hline & 8 & 1.9 & 2.3 & 11.9 & 2.1 & 2.1 & 2.1 & 0.2 & & 97.9 & 97.9 & 97.9 & 97.9 & 97.9 & 97.9 & 8 & JF422010.1 \\
\hline & 9 & 0.2 & 0.6 & 0.2 & 0.4 & 0.4 & 0.4 & 2.0 & 2.2 & & 100.0 & 97.9 & 100.0 & 100.0 & 100.0 & 9 & JF421993.1 \\
\hline & 10 & 0.2 & 0.6 & 0.2 & 0.4 & 0.4 & 0.4 & 2.0 & 2.2 & 0.0 & & 100.0 & 100.0 & 100.0 & 100.0 & 10 & JF421994.1 \\
\hline & ii & 0.2 & 0.6 & 0.2 & 0.4 & 0.4 & 0.4 & 2.0 & 2.2 & 0.0 & 0.0 & & 100.0 & 100.0 & 100.0 & 11 & JF421997.1 \\
\hline & 12 & 0.2 & 0.6 & 0.2 & 0.4 & 0.4 & 0.4 & 2.0 & 2.2 & 0.0 & 0.0 & 0.0 & & 100.0 & 100.0 & 12 & JF422013.1 \\
\hline & 13 & 0.2 & 0.6 & 0.2 & 0.4 & 0.4 & 0.4 & 2.0 & 2.2 & 0.0 & 0.0 & 0.0 & 0.0 & & 100.0 & 13 & JF421986.1 \\
\hline & 14 & 0.2 & 0.6 & 0.2 & 0.4 & 0.4 & 0.4 & 2.0 & 22 & 0.0 & 0.0 & 0.0 & 0.0 & 0.0 & & 14 & JF422012.1 \\
\hline & & i & 2 & & 4 & & 6 & & & & 10 & 11 & 12 & 13 & 14 & & \\
\hline
\end{tabular}

FIGURE 4: Homology comparison of nucleotide sequences of the isolates strains with previously reported strains (100\%).

Currently studies have showed that the mitochondrial genome structure of Tapeworms is similar to other mitochondrial genomes of eukaryotes that include 12 genes encoding proteins [17]. Among them, the mitochondrial gene cytochrome oxidase subunit 2 was the ideal genetic markers, which was used to make phylogenetic analysis [18]. In our study, the isolated parasite was phylogenetically compared to reference parasite, based on the COX2 gene coding sequence (CDS). The phylogenetic analysis demonstrated that the isolates share a close homology with some reference strains isolated in Gansu, Hunan, and Sichuan. In addition, the nucleotide sequences of the isolated strains shared $97.7 \%-100 \%$ sequence identify with the reference strains.

In conclusion, the present survey revealed the high prevalence of Cysticercus tenuicollis infection in Tibetan pigs; herein, we suggest some measures to prevent and control this infection. At first, anthelmintics must be an important practice to prevent the infection and minimize the serious economic losses. Secondly, it is important to improve the management of swine breeding to reduce the spread of disease through direct contact between dogs and Tibetan pigs. Additionally, practice of undercooked animal innards to dogs in study area should be avoided. Finally, it is necessary to change the husbandry of free-range into captivity system in these rural areas.

\section{Conflicts of Interest}

The authors declare that they have no conflicts of interest.

\section{Acknowledgments}

This study was supported by Key Science Fund of Science and Technology Agency of Tibet Autonomous Region and projects in the National Science \& Technology Pillar Program during the 12th Five-year Plan Period (2012BAD3B03) and the Chinese Agricultural Research Systems (CARS-38).

\section{References}

[1] S. N. Parmeter, D. D. Heath, and H. Twaalfhoven, "Effect of population density on growth and development of taenia hydatigena in dogs," Research in Veterinary Science, vol. 30, pp. 257259, 1987. 
[2] R. C. A. Thompson and A. J. Lymbery, Echinococcus and Hydatid Disease, CAB International, Wallingford, UK, 1995.

[3] W. Samuel and G. G. Zewde, "Prevalence, risk factors, and distribution of Cysticercus tenuicollis in visceral organs of slaughtered sheep and goats in central Ethiopia," Tropical Animal Health and Production, vol. 42, no. 6, pp. 1049-1051, 2010.

[4] J. Ma, S. W. He, H. Li et al., "First survey of helminths in adult goats in Hunan province, China," Tropical Biomedicine, vol. 31, pp. 261-269, 2014.

[5] M. Radfar, H. S. Tajalli, and M. Jalalzadeh, "Prevalence and morphological characterization of Cysticercus tenuicollis (Taenia hydatigena cysticerci) from sheep and goats in Iran," Veterinary Archives, vol. 75, pp. 469-476, 2005.

[6] B. B. Singh, R. Sharma, J. P. S. Gill, and J. K. Sharma, "Prevalence and morphological characterisation of Cysticercus tenuicollis (Taenia hydatigena cysts) in sheep and goat from north India," Journal of Parasitic Diseases, vol. 39, no. 1, pp. 80-84, 2015.

[7] M. M. Sissay, A. Uggla, and P. J. Waller, "Prevalence and seasonal incidence of larval and adult cestode infections of sheep and goats in Eastern Ethiopia," Tropical Animal Health and Production, vol. 40, no. 6, pp. 387-394, 2008.

[8] K. Blazek, J. Schramlova, and D. Hulinska, "Pathology of the migration phase of Taenia hydatigena (Palas 1766) larvae," Folia Parasitologica, vol. 32, pp. 127-137, 1985.

[9] X. J. Wang, D. S. Luo, and Y. Liu, "Prevalence of Cysticercus tenuicollis in pigs in Changsha," Hunan Journal of Animal Veterinary Science, vol. 14, pp. 22-24, 2010 (Chinese).

[10] C. L. Liu and Z. J. Lin, "The survey of Cysticercus tenuicollis in pigs," Livestock and Poultry Industry, vol. 11, pp. 50-51, 2011 (Chinese).

[11] W. P. Meng and L. B. Feng, "The investigation of Cysticercus tenuicollis in slaughtered pigs in Luxi County," Guizhou Journal of Animal Husbandry Veterinary Medicine, vol. 35, pp. 37-38, 2014 (Chinese).

[12] G. Y. Hao, G. Y. Yang, X. B. Gu et al., "Phylogenetic relationship analysis of 33 isolate of Cysticercus tenuicollis based on the mitochondrial COII gene sequences," Chinese Veterinary Science, vol. 41, pp. 684-690, 2011 (Chinese).

[13] H. Zhang, M. U. Rehman, K. Li et al., "Antimicrobial resistance of Escherichia Coli isolated from tibetan piglets suffering from white score diarrhea," Pakistan Veterinary Journal, vol. 37, no. 1, pp. 43-46, 2017.

[14] G. Q. Jiao, "Prevalence of Cysticercus tenuicillis in pigs in 5 counties, Dali," Yunnan Animal Husbandry Veterinary, vol. 6, p. 10, 2011 (Chinese).

[15] Á. M. García-González, J. E. Pérez-Martín, J. A. Gamito-Santos, R. Calero-Bernal, M. A. Alonso, and E. M. F. Carrión, “Epidemiologic study of lung parasites (metastrongylus spp.) in wild boar (sus scrofa) in southwestern Spain," Journal of Wildlife Diseases, vol. 49, no. 1, pp. 157-162, 2013.

[16] N.-Z. Zhang, D.-H. Zhou, S.-Y. Huang et al., "Seroprevalence and risk factors associated with Haemophilus parasuis infection in Tibetan pigs in Tibet," Acta Tropica, vol. 132, no. 1, pp. 94-97, 2014.

[17] H.-K. Jeon, K.-H. Kim, and K. S. Eom, "Complete sequence of the mitochondrial genome of Taenia saginata: comparison with T. solium and T. asiatica," Parasitology International, vol. 56, no. 3, pp. 243-246, 2007.

[18] W.-Z. Jia, H.-B. Yan, A.-J. Guo et al., "Complete mitochondrial genomes of Taenia multiceps, T. hydatigena and T. pisiformis: additional molecular markers for a tapeworm genus of human and animal health significance," BMC Genomics, vol. 11, no. 1, article 447, 2010. 

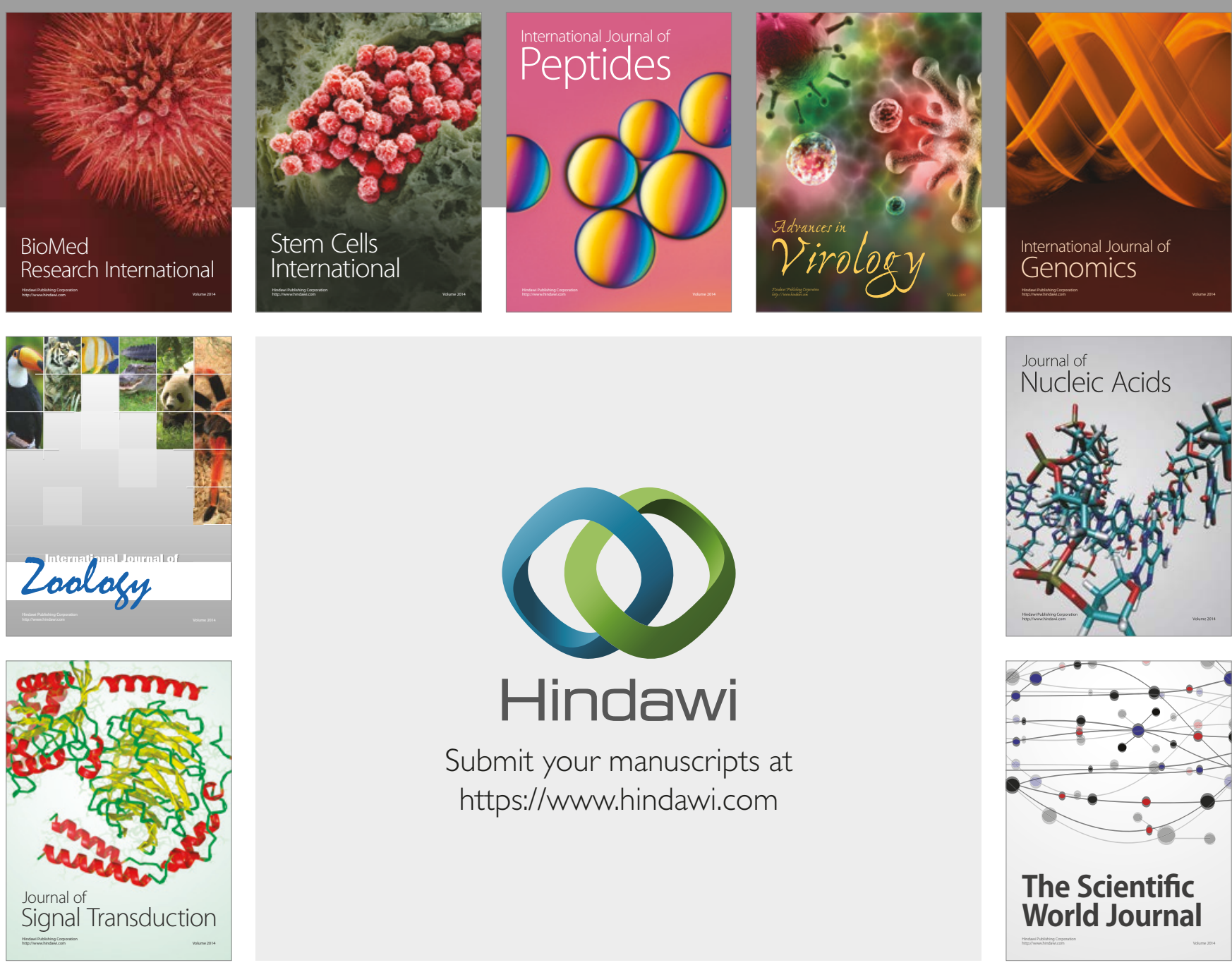

Submit your manuscripts at

https://www.hindawi.com
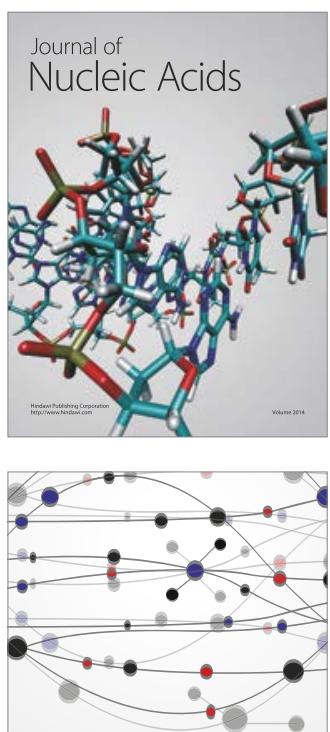

The Scientific World Journal

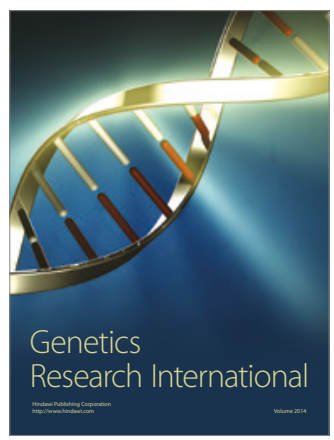

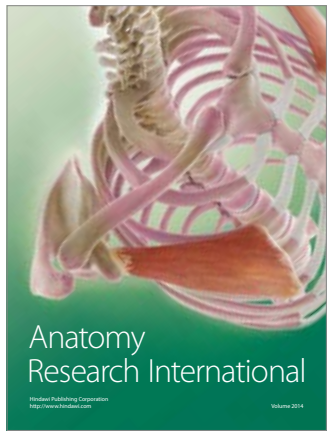

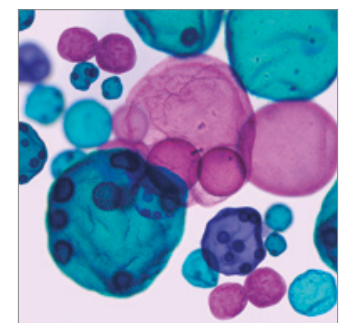

International Journal of Microbiology
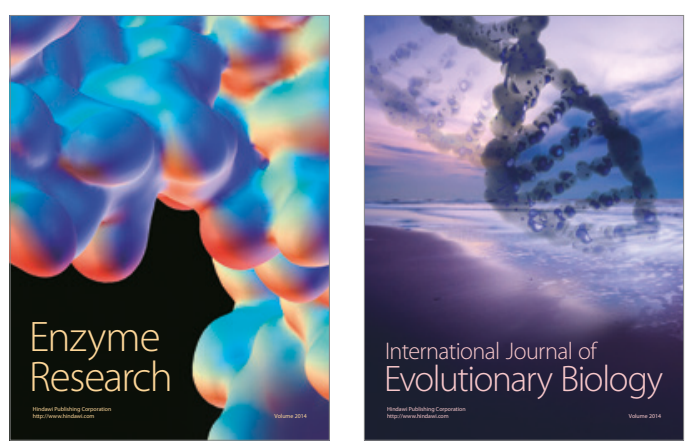
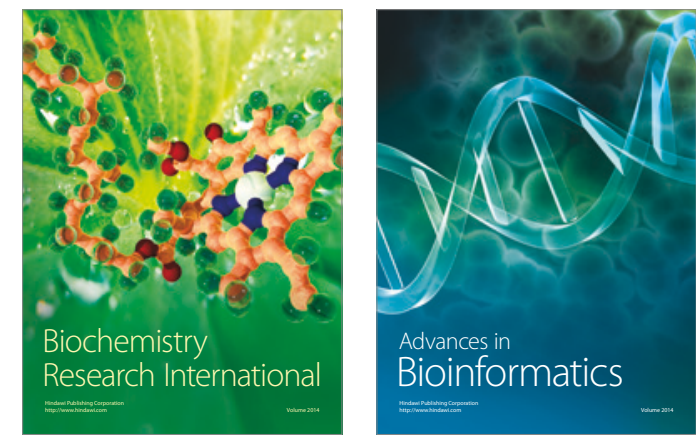

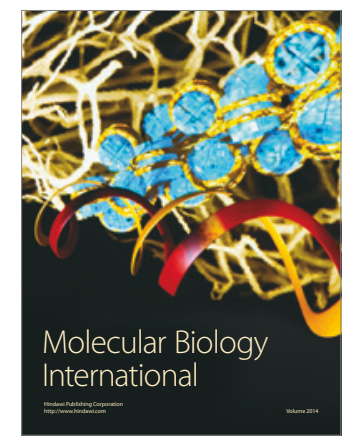

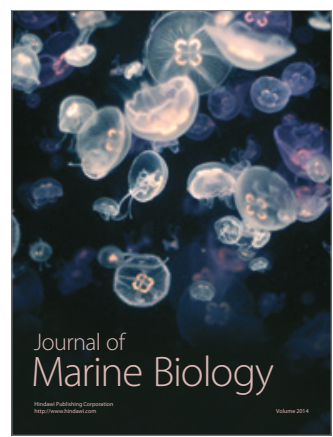

